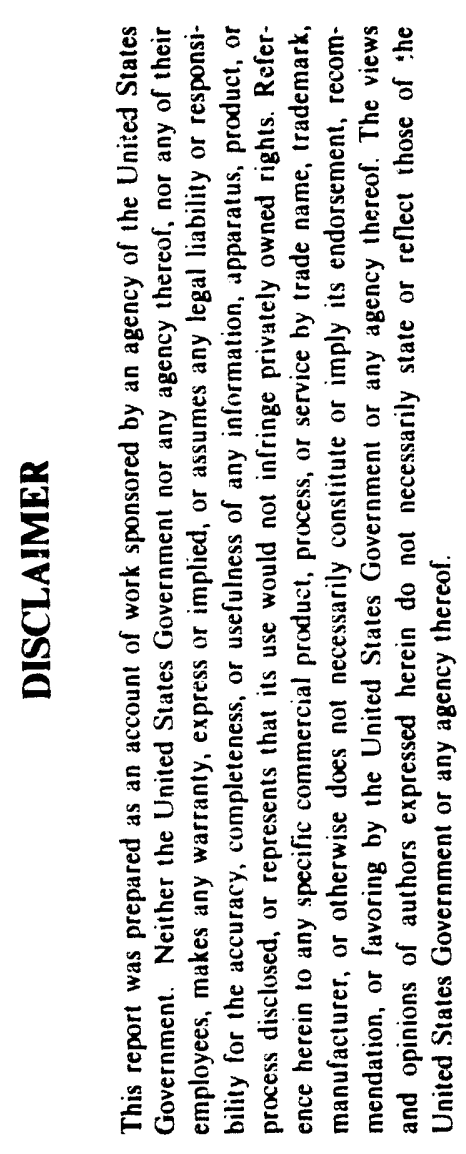

\title{
TIME OPTIMAL TRAJECTORIES FOR A TWO WHEELED ROBOT*
}

CONF-920540--8

DE92 000069

\author{
D. B. Reister and F. G. Pin \\ Center for Engineering Systems Advanced Research \\ Oak Ridge National Laboratory \\ P.O. Box 2008 \\ Building 6025, MS-6364 \\ Oak Ridge, TN 37831-6364
}

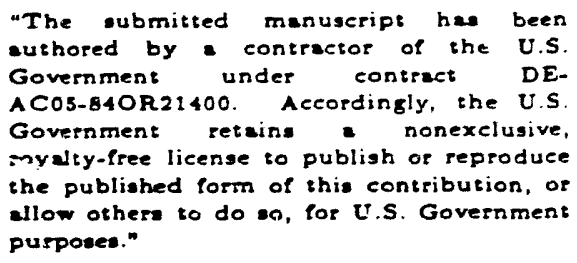

Contact: D. B. Reister, Oak Ridge National Laboratory, P.O. Box 2008, Bldg. 6025, MS-6364, Oak Ridge, TN 37831-6364, U.S.A., Telephone: (615)574-2272

Submitted to: The 1992 IEEE International Conference on Robotics and Automation, Nice, France, May 10-15, 1992

Research sponsored by the Office of Engineering Research Program, Basic Energy Sciences, of the U.S. Department of Energy, under contract No. DE-AC05-84OR21400 with Martin Marietta Energy Systems, Inc. 


\title{
TIME OPTIMAL TRAJECTORIES FOR A TWO WHEELED ROBOT
}

\author{
D. B. Reister and F. G. Pin \\ Center for Engineering Systems Advanced Research \\ Oak Ridge National Laboratory \\ P.O. Box 2008 \\ Oak Ridge, TN 37831-6364
}

\begin{abstract}
This paper addresses the problem of time-optimal motions for a mobile platform in a 2-D planar environment. The platform is assumed to be of the skid-steer type, with two non-steerable independently driven wheels. The overall mission of the robot is expressed in terms of a sequence of via points at which the platform must be at rest in a given configuration (position and orientation). The objective is to plan time-optimal trajectories between these configurations assuming an unobstructed environment.

Using Pontryagin's maximum principle, we formally demonstrate that the time optimal motions of the platform are bang-bang (at each instant, the acceleration on each wheel is either at the upper or lower limit). The optimal trajectories can be characterized by a set of unique parameters corresponding to the switch times (the times at which the wheel accelerations change sign). We show numerically that trajectories with three switch times (two on one wheel, one on the other) can reach any position, while trajectories with four switch times can reach any configuration. Given the desired final configuration of the platform, we can search the parameter space and find the switch times that will produce particular paths to the configuration. We show numerically that a uniquely defined subset of these paths are time optimal by calculating the dual variables required by the maximum principle.
\end{abstract}




\section{INTRODUCTION}

A variety of designs have been implemented for mobile robots. These can be classified in three major categories: 1 . omnidirectional platforms, that utilize steerable wheels (see [1]. [2] . [3]), roller-equipped wheels (see [4]) or orthogonal wheel assemblies (see [5]): 2. carlike platforms that incorporate controlled steerable wheels on one axle and non-steerable wheels on another axle (see [6]); and 3. skid-steer platforms that include two non-steerable independently driven wheels (see [7], [8], [9]). This paper is concerned with shid-steer platforms. A typical mission for a mobile robot can be described by a sequence of ria points at which the robot comes to rest in a given configuration (position and orientation) to perform a given task (manipulation, sensing, etc.). The problem considered in this paper is that of finding time-optimal motions of the robot in Cartesian space and the corresponding control trajectories that will move the robot from an initial configuration to a final configuration in an unobstructed environment.

The proposed approach to find the control trajectories that lead to time-optimal motions of the platform involves utili ation of Pontryagin's maximum principle ([10]). A variety of authors have applied the maximum principle to the optimal motion planning of serial-link manipulators [11],[12],[13],[14],[15],[16],[17]. For the time optimal motion of a manipulator with bounded control torques, the controls occur linearly in the Hamiltonian and the optimal values of the controls are determined by the dual variables. When its dual variable is not zero, the optimal control is bang-bang (the optimal control is at its upper limit for a positive dual variable and at its lower limit for a negative dual variable). When its dual variable is zero for a finite interval, the optimal control is singular and will be in the region between the bounds. An important issue is to determine when the optimal solution is bang-bang and when it is singular. A variety of authors [18],[19],[20],[21] have addressed this issue and proven that there cannot be a finite time interval when the optimal control for a manipulator is singular for all the control torques. In other words, at least one of the controls is always bang-bang. Further work [22] led to the determination of the parameter values for singular solutions for three types of manipulators: cylindrical, spherical, and two link planar. It has been demonstrated [23],[24], that the singular solution for the cylindrical case is not optimal.

In the following sections, we present the equations of motion for a skid-stess type of platform. We then use the maximum principle to derive the conditions for time-optinal motions of the platform and demonstrate that the optimal controls are always bangbang. Using this result, we show that, for a system with bounded wheel accelerations, control trajectories with three switch times (times at which one of the wheel's acceleration 
changes sign) allow the robot to reach any point in cartesian space while with four switch times, the robot can reach any configuration. The solutions in those cases can be simply parameterized in terms of the control switch times. Using a non-linear search algorithm in the parameter space, we can directly obtain some uniquely defined three-switch-times and four-switch-times control trajectories for any desired final configuration of the robot. We then show numerically that these paths are time-optimal by calculating the dual variables required by the maximum principle.

\section{KINEMATIC EQUATIONS OF MOTION FOR THE PLATFORM}

A skid-steer type of platform must satisfy nonholonomic constraints and cannot follow an arbitrary path through configuration space. In this section, we will present a kinematic model of the platform. We will assume that the wheels do not slip and that the wheel accelerations can instantaneously switch from their upper limit to their lower limit. The configuration of the platform is described by three coordinates: the Cartesian coordinates ( $x$ and $y$ ) of the midpoint of the wheel axle and the orientation of the platform with respect to the $x$ axis $(\phi)$ (see Fig. 1). The joint variables are denoted by $\Theta_{R}$ and $\Theta_{L}$, representing the rotation times the radius of the right and left wheels, and are measured in meters. The wheel velocities are denoted by $\omega_{\mathrm{R}}$ and $\omega_{\mathrm{L}}$ and are measured in meters per second. The control variables are the right and left wheel accelerations $\left(u_{R}\right.$ and $u_{L}$ ). The kinematic model links the Cartesian variables to the control variables through the wheel velocities:

$$
\begin{gathered}
\dot{\phi}=\left(\omega_{\mathrm{R}}-\omega_{\mathrm{L}}\right) / \mathrm{D} \\
\dot{\mathrm{x}}=\left(\omega_{\mathrm{R}}+\omega_{\mathrm{L}}\right) \cos (\phi) / 2 \\
\dot{\mathrm{y}}=\left(\omega_{\mathrm{R}}+\omega_{\mathrm{L}}\right) \sin (\phi) / 2 \\
\dot{\omega}_{\mathrm{R}}=\mathrm{u}_{\mathrm{R}} \\
\dot{\omega}_{\mathrm{L}}=\mathrm{u}_{\mathrm{L}} \\
\dot{\Theta}_{\mathrm{R}}=\omega_{\mathrm{R}}
\end{gathered}
$$




$$
\dot{\Theta}_{\mathrm{L}}=\omega_{\mathrm{L}}
$$

where $D$ is the distance between the centers of the wheels.

Given a trajectory for the control variables, Eqs. (1) through (7) can be integrated to determine the Cartesian variabies and the joint variables. For a non-holonomic system, the time sequence of the controls is necessary to determine the final position. For example, if both wheels move a meter, the platform will move forward a meter. If the right wheel moves a meter and then the left wheel moves a meter, the platform will move less than a meter forward and will have moved to the left. Although the final values for the joint variables and the orientation are the same for the two maneuvers, the final values for the position are not the same.

\section{THE GENERAL FORM OF THE OPTIMAL CONTROLS}

Since its discovery in 1956, the Pontryagin maximum principle [10] has been used to solve a wide variety of optimization problems. In this section we utilize the maximum principle to derive the conditions for time optimality of the platform motion between the initial and final configurations. For our problem, we can define five state variables: $\mathrm{x}_{1}=\mathrm{x}, \mathrm{x}_{2}=\mathrm{y}, \mathrm{x}_{3}=\phi, \mathrm{x}_{4}=\omega_{\mathrm{R}}$, and $\mathrm{x}_{5}=\omega_{\mathrm{L}}$ and rewrite the equations of motion (1) to (5) as:

$$
\begin{gathered}
\dot{x}_{1}=f_{1}(x, u)=\left(x_{4}+x_{5} \cdot \cos x_{3} / 2\right. \\
\dot{x}_{2}=f_{2}(x, u)=\left(x_{4}+x_{5}\right) \sin x_{3} / 2 \\
\dot{x}_{3}=f_{3}(x, u)=\left(x_{4}-x_{5}\right) / D \\
\dot{x}_{4}=f_{4}(x, u)=u_{1} \\
\dot{x}_{5}=f_{5}(x, u)=u_{2}
\end{gathered}
$$

where the two control variables are: $u_{1}=u_{R}$ and $u_{2}=u_{L}$.

In vector notation, the equations of motion for the state vector $\mathrm{x}$ are:

$$
\dot{\mathrm{x}}=\mathrm{f}(\mathrm{x}, \mathrm{u})
$$


ard the optimization problem is to find a control vector [u] that will move the system from the initial state $x^{0}$ to the final state $x^{1}$ while minimizing an objective functional. For our case, the goal is to minimize the transition time, assuming that the wheel accelerations are bounded: $\left|\mathrm{u}_{1}\right| \leq \mathrm{a}_{\max }$ and $\left|\mathrm{u}_{2}\right| \leq \mathrm{a}_{\max }$.

Pontryagin introduces a system of dual variables $[\psi]$ that satisfy:

$$
\dot{\psi}_{\mathrm{i}}=-\sum_{\mathrm{j}=1}^{\mathrm{n}} \frac{\partial \mathrm{f}_{\mathrm{j}}(\mathrm{x}, \mathrm{u})}{\partial \mathrm{x}_{\mathrm{i}}} \psi_{\mathrm{j}}, \quad \mathrm{i}=1,2, \ldots, \mathrm{n}
$$

with initial conditions:

$$
\psi_{\mathrm{i}}\left(\mathrm{t}_{\mathrm{o}}\right)=\lambda_{\mathrm{i}} \quad \mathrm{i}=1,2, \ldots, \mathrm{n},
$$

where, in our problem, $\mathrm{n}=5$.

Equations (14) are linear and homogeneous and have a unique solution for given boundary conditions. If the equations of motion and the dual variables are combined into a single Hamiltonian $[\mathrm{H}]$ :

$$
\mathrm{H}(\psi, \mathrm{x}, \mathrm{u})=\sum_{\mathrm{j}=1}^{\mathrm{n}} \psi_{\mathrm{j}} \mathrm{f}_{\mathrm{j}}(\mathrm{x}, \mathrm{u})
$$

then the maximum principle states that (see Theorem 2 of Ref. [10]), if $u$ is an admissible control that transfers the phase point from the initial state $x\left(t_{0}\right)=x^{0}$ to the goal state $x^{1}=x\left(t_{1}\right)$ at some time $t_{1}$, then $u(t)$ and the trajectory $x(t)$ are time-optimal if there exists a non-zero continous vector function $\left(\psi_{1}(t), \ldots \psi_{n}(t)\right)$ defined by Eq. (14), and such that $u(t)$ maximizes the function $H$ for all $t, t_{0} \leq t \leq t_{1}$.

For our mobile platform problem defined by Eqs. (8) through (12), the Hamiltonian function $\mathrm{H}$ is:

$$
\mathrm{H}(\psi, \mathrm{x}, \mathrm{u})=\psi_{1} \mathrm{f}_{1}+\psi_{2} \mathrm{f}_{2}+\psi_{3} \mathrm{f}_{3}+\psi_{4} \mathrm{u}_{1}+\psi_{5} \mathrm{u}_{2}
$$

where the functions $f_{i}(x, u)$ are defined by Eqs. (8) to (12). The equations for the dual variables are:

$$
\begin{gathered}
\dot{\psi}_{1}=0 \\
\dot{\psi}_{2}=0 \\
\dot{\psi}_{3}=g\left(x_{3}\right)\left(x_{4}+x_{5}\right)
\end{gathered}
$$




$$
\begin{aligned}
& \dot{\psi}_{4}=-\mathrm{g}^{\prime}\left(\mathrm{x}_{3}\right)-\dot{\psi}_{3} / \mathrm{D} \\
& \dot{\psi}_{5}=-\mathrm{g}^{\prime}\left(\mathrm{x}_{3}\right)+\psi_{3} / \mathrm{D}
\end{aligned}
$$

where $g\left(x_{3}\right)$ and $g^{\prime}\left(x_{3}\right)$ are defined by:

$$
\begin{gathered}
\mathrm{g}\left(\mathrm{x}_{3}\right)=\left(\psi_{1} \sin \mathrm{x}_{3}-\psi_{2} \cos \mathrm{x}_{3}\right) / 2 \\
\mathrm{~g}^{\prime}\left(\mathrm{x}_{3}\right)=\frac{\mathrm{dg}\left(\mathrm{x}_{3}\right)}{\mathrm{dx_{3 }}}=\left(\psi_{1} \cos \mathrm{x}_{3}+\psi_{2} \sin \mathrm{x}_{3}\right) / 2
\end{gathered}
$$

The platform moves from an arbitrary initial configuration to a final configuration. We can choose the coordinate system such that the initial position is $(0,0)$ and the initial orientation is $\phi=0$. The boundary conditions corresponding to the robot being at rest at the initial state $x^{0}=x\left(t_{0}\right)$ and final state $x^{1}=x\left(t_{1}\right)$, are

$$
\begin{aligned}
& x\left(t_{0}\right)=(0,0,0,0,0) \\
& x\left(t_{1}\right)=(x, y, \phi, 0,0)
\end{aligned}
$$

When the final state is a configuration, the final values of the dual variables are arbitrary. When the final state is not fully specified, the transversality condition determines the final values of the dual variables (see Theorem 3 of Ref. [10]). For example, when the final state is a position (and the final orientation is not specified), the final value of the third dual variable $\left(\psi_{3}\right)$ is zero.

The optimal values for the control variables $\left(u_{1}\right.$ and $u_{2}$ ) are those which maximize the Hamiltonian. It is clear that when the dual variables $\psi_{4}$ and $\psi_{5}$ are not zero, the optimal control is bang-bang; when $\psi_{4}$ is positive, $\mathrm{u}_{1}=\mathrm{a}_{\max }$ and when $\psi_{4}$ is negative, $\mathrm{u}_{1}=-\mathrm{a}_{\max }$, and similarly for $\psi_{5}$ and $u_{2}$. If $\psi_{4}$ or $\psi_{5}$ is zero for a finite time interval, the optimal control becomes singular. In the remaining of this section we will prove that all possible optimal solutions of the system are bang-bang.

\section{Theorem}

All optimal control solutions for the system defined by Eqs. (8) through (12), (25) and (26) are bang-bang. 
Proof

From Eq. (17), we know that when the dual variables $\psi_{4}$ and $\psi_{5}$ are not zero, the optimal control is bang-bang: and when $\psi_{4}$ or $\psi_{5}$ is zero for a finite interval, the optimal control is singular. To prove the theorem, we will investigate all the singular solutions and show that they are either impossible or bang-bang.

When the optimal control is singular, $\psi_{4}$ (or $\psi_{5}$ ) is zero for a finite interval. Since the equations for $\psi_{4}$ and $\psi_{5}$ have the same structure, we will only consider the case where $\psi_{4}$ is zero for a finite interval. If $\psi_{4}$ is zero for a finite time interval, $\left[t_{2}, t_{3}\right]$, all of its derivatives are also zero on the interval and Eq. (21) yields:

$$
\psi_{3}=-\mathrm{Dg}^{\prime}\left(\mathrm{x}_{3}\right)
$$

The time derivative of Eq. (27) implies:

$$
\dot{\psi}_{3}=-\mathrm{Dg}^{\prime \prime}\left(\mathrm{x}_{3}\right) \dot{\mathrm{x}}_{3}=\mathrm{g}\left(\mathrm{x}_{3}\right)\left(\mathrm{x}_{4}-\mathrm{x}_{5}\right)
$$

where we have used the fact that $g^{\prime \prime}=-g$.

Since the right sides of Eqs. (20) and (28) must be equal, we have:

$$
g\left(x_{3}\right) x_{5}=0
$$

This leads to two general cases:

Case 1. $g\left(x_{3}\right)=0$

Case 2. $\mathrm{x}_{5}=0$

Case 1 can be subdivided into four subcases:

Case 1.1. $\tan \mathrm{x}_{3}=\psi_{2} / \psi_{1}, \quad \psi_{1} \neq 0, \quad \psi_{2} \neq 0$

Case 1.2. $\cos \mathrm{x}_{3}=0 \quad \psi_{1}=0, \quad \psi_{2} \neq 0$

Case 1.3. $\sin \mathrm{x}_{3}=0 \quad \psi_{1} \neq 0, \quad \psi_{2}=0$

Case 1.4. $\psi_{1}=\psi_{2}=0$ 
Consider cases $1.1,1.2$, and 1.3

Since $\psi_{1}$ and $\psi_{2}$ are constant (from Eqs. (18) and (19)) and $g\left(x_{3}\right)=0, x_{3}$ is a constant and $\dot{x}_{3}=0$ on the finite interval $\left[t_{2}, t_{3}\right]$. Thus, these cases correspond to the robot moring along a straight line over a finite interval of time, (the angle $\phi=x_{3}$ given by Eqs. (32), (33), or (34)) and Eq. (10) requires that $x_{4}=x_{5}$, i.e., that the controls on both wheels be equal, $\mathrm{u}_{1}=\dot{\mathrm{x}}_{4}=\dot{\mathrm{x}}_{5}=\mathrm{u}_{2}$, over the interval $\left[\mathrm{t}_{2}, \mathrm{t}_{3}\right]$. Now, since $\mathrm{g}=0, \dot{\psi}_{3}=0$ (using Eq. (20)), and $\psi_{3}$ is a constant. Since $\mathrm{g}^{\prime}$ is also a constant $\left(\psi_{1}, \psi_{2}\right.$, and $\mathrm{x}_{3}$ are constants in Eq. (24)), $\dot{\psi}_{5}$ is constant (from Eq. (22)).

If $\dot{\psi}_{5}$ is a non-zero constant, then $\psi_{5}$ varies linearly, and the optimal controls $u_{2}$ and $u_{1}$ are equal and bang-bang over the interval $\left[t_{2}, t_{3}\right]$. If $\dot{\psi}_{5}$ is zero, then Eq. (22) yields $\psi_{3}=\operatorname{Dg}^{\prime}\left(\mathrm{x}_{3}\right)$ which, with Eq. (27), implies $\psi_{3}=\mathrm{g}^{\prime}\left(\mathrm{x}_{3}\right)=0$. If both $\mathrm{g}=0$ and $\mathrm{g}^{\prime}=0$, then $\psi_{1}=\psi_{2}=0$. This violates the assumptions in Eqs. (32), (33), or (34) and consequently $\dot{\psi}_{5}=0$ is not a valid solution for these cases. Thus, for cases $1.1,1.2$, and 1.3 , the only admissible controls are bang-bang.

\section{Consider Case 1.4}

If $\psi_{1}=\psi_{2}=0$ over the interval $\left[\mathrm{t}_{2}, \mathrm{t}_{3}\right]$, they are also zero over the entire trajectory (from Eqs. (18) and (19)). Thus $g=g^{\prime}=0$ and $\psi_{3}=0$ (from Eq. (27)) over the entire trajectory. Consequently, $\dot{\psi}_{5}=0$ from Eq. (22), requiring the dual variable $\psi_{5}$ to be constant over the entire trajectory. This is not an admissible case for our problem since the corresponding extremal controls $u_{2}$ would not change sign over the entire trajectory, leading to a linearly increasing or decreasing wheel velocity and making Eq. (26) impossible to satisfy. Thus case 1.4 does not lead to admissible controls for our problem.

\section{Consider Case 2}

If $\mathrm{x}_{5}=0$ over a finite time interval $\left[\mathrm{t}_{2}, \mathrm{t}_{3}\right]$, then the control $\mathrm{u}_{2}=\dot{\mathrm{x}}_{5}=0$ over the interval, and consequently its dual variable $\psi_{5}$ and its derivative $\dot{\psi}_{5}$ must be zero over the entire finite interval. Since $\psi_{4}=\dot{\psi}_{4}=0$, Eqs. (21) and (22) require $\psi_{3}=0$ and $\mathrm{g}^{\prime}\left(\mathrm{x}_{3}\right)=0$ over the entire interval, and consequently $\dot{\psi}_{3}=0$. Equation (20) thus requires either that $x_{4}=x_{5}=0$, which is an inadmissible case (since, from Eqs. (8) through (12), no motion of the robot would take place over a finite time interval during the trajectory, which onsequently can not be time optimal), or that $\mathrm{g}\left(\mathrm{x}_{3}\right)=0$ over the finite time interval. If both $g$ and $g^{\prime}$ are zero over the finite time interval, then $\psi_{1}=\psi_{2}=0$. Since $\psi_{1}$ and $\psi_{2}$ are constant over the entire trajectory (from Eqs. (18) and (19)), they must be zero 
over the entire trajectory and Eq. (23), (24), and (20) lead to $\mathrm{g}=\mathrm{g}^{\prime}=\dot{\psi}_{3}=0$ over the entire trajectory. Consequently, since $\psi_{3}=0$ over the finite time interval and $\psi_{3}=0$ orer the entire trajectory, $\psi_{3}=0$ and $\dot{\psi}_{4}=\dot{\psi}_{5}=0$ (from Eqs. (21) and (22)) over the entire trajectory. Therefore, since $\psi_{4}=0$ and $\psi_{5}=0$ over the finite interval, they also are zero on the entire trajectory. Thus this case is not admissible since all dual variables $\psi_{\mathrm{i}}$ are zero over the entire trajectory.

Sinilar arguements demonstrate that singular solutions corresponding to the dual variable $\psi_{5}$ being zero over a finite time interval lead to optimal controls that are bang-bang, or are inadmissible. Thus, all optimal control solutions for the system defined by Eqs. (8) through (12), (25), and (26) are bang-bang.

A similar result was recently outlined in Ref. [25], however, without consideration given to cases $1.2,1.3$, and 1.4 in the demonstration.

\section{PARAMETERIZATION OF THE OPTIMAL TRAJECTORIES}

The Pontryagin Maximum Principle converts the problem of optimal path planning from a problem involving trajectories in state space and time to a static optimization problem in parameter space. Two sets of parameters are available: the initial conditions for the dual variables $\left[\lambda_{\mathbf{k}}\right]$ and the switch times. Using either set of parameters, nonlinear search techniques can be used to determine optimal paths that move from the initial state to the final state. In the remainder of this paper, we will use the switch times to parameterize the control paths. Using a conjecture that the optimal trajectories have at most four switch times, we construct the corresponding parameterized trajectory space. For this we numerically integrate the control paths to calculate the Cartesian paths and some auxilary variables. We then use the auxilary variables to calculate the initial conditions for the dual variables, numerically integrate to calculate the dual variables, and verify that the selected Cartesian paths are optimal by showing that a corresponding set of dual variables exists that verify the conditions of the Pontryagin Maximum Principle and is consistent with the control paths. In this section, we will parameterize the control paths, define the auxilary variables, and outline the procedure used to calculate the initial conditions for the dual variables.

We have proven that the optimal controls are bang-bang. Thus, each wheel is always either accelerating or decellerating at the maximum rate $\left(a_{\max }\right)$, and the wheel velocity trajectories consist of successive segments of linearly increasing or decreasing velocity. The wheel acceleration changes sign at a switch time. We can characterize a control trajectory 
by the number of switch times. The path with the smallest number of switch times has two (one for each wheel). However, there are only two paths with two switch times: translation and rotation. Bang-bang type paths typically utilized for skid-steer robots include a rotation followed by a translation which can reach any position and requires five switch times, or a rotation, translation, rotation which can reach any configuration and has eight switch times. We will show that a platform path involving three switch times (two on one wheel, one on the other) allows the robot to reach any position, while a path with four switch times (either two for each wheel or one for one wheel and three for the other) allows it to reach any configuration. First, we derive an analytical expression for the rotation of a wheel with three switch times. By adjustir. g parameters, the expression will yield the rotation for motions with one or two switch times. We assume that the initial value for the wheel rotation $(\Theta)$ is zero. The initial and final values for the wheel velocity are zero, thus, during half of the trajectory, the wheel will accelerate and during the other half it will decellerate. Consequently, if $\mathrm{T}$ is half of the time for the trajectory and $\tau_{1}$ and $\tau_{1}+\tau_{2}$ denote the first two switch times, the third switch time is automatically determined (the trajectory will have two periods of positive acceleration of length $\tau_{1}$ and $\mathrm{T}-\tau_{1}$, interleaved by two periods of negative acceleration of length $\tau_{2}$ and $T-\tau_{2}$ ). We assume that the acceleration is given by: $u$ for $\left[0, \tau_{1}\right],-u$ for $\left[\tau_{1}, \tau_{1}+\tau_{2}\right], u$ for $\left[\tau_{1}+\tau_{2}, \tau_{2}+\mathrm{T}\right]$, and $-u$ for $\left[\tau_{2}+T, 2 T\right]$. Integrating Eqs. (4) and (6), we can calculate the final value for the wheel rotation for a three switch time trajectory of the wheel:

$$
\Theta(2 \mathrm{~T})=\mathrm{u}\left[2 \tau_{1} \tau_{2}-2 \tau_{2} \mathrm{~T}+\mathrm{T}^{2}\right]
$$

When $\tau_{2}=\mathrm{T}$, the wheel trajectory reduces to one with two switch times and when $\tau_{1}=\mathrm{T}$ and $\tau_{2}=\mathrm{T}$ the wheel trajectory reduces to one with one switch time. The corresponding wheel rotations are:

$$
\begin{gathered}
\Theta(2 \mathrm{~T})=\mathrm{uT}\left[2 \tau_{1}-\mathrm{T}\right] \quad \text { when } \tau_{2}=\mathrm{T} \\
\Theta(2 \mathrm{~T})=\mathrm{u}\left[\mathrm{T}^{2}\right] \quad \text { when } \tau_{1}=\mathrm{T} \text { and } \quad \tau_{2}=\mathrm{T}
\end{gathered}
$$




\subsection{PARAMETERIZATION OF BANG-BANG TRAJECTORIES WITH GIVEN FINAL ORIENTATION}

While the equations of motion for the Cartesian variables for position $(x, y)$ do not have general analytical solutions, we can derive an analytical solution for the Cartesian variable for orientation ( $\phi)$. Using Eqs. (6) and (7), Eq. (1) may be written:

$$
\dot{\phi}=\left(\dot{\Theta}_{R}-\dot{\Theta}_{L}\right) / D
$$

If the initial conditions for the wheel rotation joint variables are zero, Eq. (39) can be integrated to yield:

$$
\phi=\phi_{0}+\left(\Theta_{R}-\Theta_{L}\right) / D
$$

where $\phi_{0}$ is the initial value of the orientation.

Equations (36), (37), and (38) relates the switch times to the wheel rotation for cases where the wheel controls include three, two or one switch times respectively, while Eq. (40) relates the wheel rotation to the change in orientation. Thus, given switch times for both wheels, we can calculate the final change in orientation. Alternatively, given the desired final change in orientation, we have a constraint on the switch times of both wheels which allows us to eliminate one parameter in the parameterization of the platform path. Thus, when the final orientation is specified, a bang-bang-type platform path involving four switch times can be parameterized with two parameters $\left(\tau_{1}\right.$ and $\left.T\right)$. Note that with no orientation specified, a bang-bang-type platform path involving three switch times can also be parameterized with two parameters.

\subsection{CONSIDERATIONS ABOUT THE NUMBER OF SWITCH TIMES}

To verify that a given bang-bang trajectory is optimal, its associated dual variables must be found as non-zero continuous functions, consistent with the control paths (e.g., $\mathrm{u}_{1}$ is positive when $\psi_{4}$ is positive, etc). The dual variables satisfy Eqs. (18) to (22), with initial conditions given by Eq. (15). Since this set of equations is linear and homogeneous, it has a unique solution for given boundary conditions $\lambda_{i}$. Thus, to ensure that an appropriate set of dual variables can be found, a feasible set of $\lambda_{\mathrm{i}}$ must exist. From Eqs. (18) and (19), the first two dual variables are constants. Thus $\psi_{1}=\lambda_{1}$ and $\psi_{2}=\lambda_{2}$. Using Eqs. (8), (9), (20), and (23), the third dual variable satisfies: 


$$
\dot{\psi}_{3}=\psi_{1} \dot{x}_{2}-\psi_{2} \dot{x}_{1}
$$

Since the initial position is $(0,0)$. Eq. (41) can be integrated:

$$
\psi_{3}=\lambda_{1} x_{2}-\lambda_{2} x_{1}+\lambda_{3}
$$

The fourth and fiith dual variables satisfy Eqs. (21) and (22). The right sides of these equations depend on the first three state variables. To integrate the equations, we define four auxilary variables $\left(\mathbf{z}_{\mathbf{i}}\right)$ by:

$$
\begin{array}{cc}
\dot{z}_{1}=\sin \left(\mathrm{x}_{3}\right) & \mathrm{z}_{1}(0)=0 \\
\dot{\mathrm{z}}_{2}=\cos \left(\mathrm{x}_{3}\right) & \mathrm{z}_{2}(0)=0 \\
\dot{z}_{3}=\mathrm{x}_{1} & \mathrm{z}_{3}(0)=0 \\
\dot{z}_{4}=\mathrm{x}_{2} & \mathrm{z}_{4}(0)=0
\end{array}
$$

Using the atxilary variab!=, the solutions for the fourth and fifth dual variables are:

$$
\begin{gathered}
\psi_{4}=-\lambda_{1}\left(\mathrm{z}_{2} / 2+\mathrm{z}_{4} / \mathrm{D}\right)+\lambda_{2}\left(\mathrm{z}_{3} / \mathrm{D}-\mathrm{z}_{1} / 2\right)-\lambda_{3}(\mathrm{t} / \mathrm{D})+\lambda_{4} \\
\psi_{5}=\lambda_{1}\left(\mathrm{z}_{4} / \mathrm{D}-\mathrm{z}_{2} / 2\right)-\lambda_{2}\left(\mathrm{z}_{1} / 2+\mathrm{z}_{3} / \mathrm{D}\right)+\lambda_{3}(\mathrm{t} / \mathrm{D})+\lambda_{5}
\end{gathered}
$$

We know that $\psi_{4}$ and $\psi_{5}$ vanish at the switch rimes, therefore, the initial conditions $\lambda_{i}$ for the dual variables can be determined by solving the matrix equation: $A \lambda=0$, where $\lambda^{T}=\left(\lambda_{1}, \ldots \lambda_{5}\right)$ and the rows of the matrix $A$ are defined by Eqs. (47) and (48) applied at the switch times. If the switch time is for the right wheel, $\psi_{4}$ is equal to zero and Eq. (47) defines a row of $\mathrm{A}$. If the switch time is for the left wheel, $\psi_{3}$ is equal to zero and Eq. (48) defines a row of $\mathrm{A}$. For a control path with $\mathrm{n}$ switch tines, the matrix thus has $\mathrm{n}$ rows. For the vector $\lambda$ to be non-zero, the rank of $A$ must be four or less. If a control path has four or less switch times, a non-zero solution is possible. If the control path has more than four switch times, either the coiumns of the $A$ mairix must be inneariy dependent 
or only the trivial solution $\lambda=0$ exists. Thus we will first explore the space of platform paths involving four switch times to investigate possible general solutions of our problem when the final orientation is specified. Similarly, when the final state is a position (and the final orientation is not specified), the tranversality condition (see Ref. 10. Theorem 3 , p. 50) requires tr:: the final value of the third dual variable be zero and Eq. (42) defines a row of $A$. Thus, when the final state is a position, a path involving three switch times would produce a four row matrix, and a non-zero solution for the vector $\lambda$ is possible. For this type of problera with no final orientation specified, we have therefore constructed the space of platform paths involving three switch times.

\section{TIME-OPTIMAL SOLUTIONS}

Our objective is to move from an arbitrary initial configuration to an arbitrary final configuration in minimum time. Each configuration is described by three coordinates $(x, y, \phi)$, where $(x, y)$ is the location of the midpoint between the two wheels and $\phi$ is the orientation of the robot. We can choose the coordinate system such that the initial configuration is $(0,0,0)$. We will assume that the final configuration is in the first quadrant. If we can reach any configuration in the first quadrant, we can reach the corresponding configuration in the other three quadrants by changing the direction of motion (symmetry about the $y$ axis) or by exchanging the trajectories for the two wheels (symmetry about the $x$ axis). The initial and final values for the wheel velocities ( $\omega_{R}$ and $\omega_{L}$ ) are zero.

Based on the considerations about numbers of switch times given in the previous section, we explore the space of platform paths that involve at most four switch times. We will find that the paths with three switch times can reach any position, while the paths with four switch times can reach any configuration. To construct the spaces of paths, we can numerically integrate the equations of motion using a fourth-order RungeKutta method [26] to determine the final positions of the platforms while varying the two parameters which parameterized the paths. To produce the figures presented below, an arbitrary value of the acceleration bound: $a_{\max }=0.5$ meters $/$ second ${ }^{2}$ has been utilized.

\subsection{TIME OPTIMAL PATHS WHEN NO FINAL ORIENTATION IS SPECIFIED}

To reach points when $n=$ final orientation is specified, path with at most three switch times, parameterized with $\left(\tau_{i}, T\right)$ are investigated. When $\tau_{i}=0$, i.e., for a path with only 
two switch times, the motion is a pure rotation or a pure translation with the platform platform moving along the $\mathrm{x}$-axis and

$$
\mathrm{x}=\mathrm{u} \mathrm{T}^{2}
$$

We now consider paths with one switch time on the right wheel and two (non-zero) for the left wheel. For a constant value of $T$, we can let the first switch time for the left wheel $\left(\tau_{1}\right)$ increase from zero to $\mathrm{T}$ and find the corresponding final locations of the platform. The curve representing these final locations of the platform starts from the point on the $x$ axis at $\mathrm{x}=\mathrm{u} \mathrm{T}^{2}$, sweeps through the first quadrant and spirals to the origin (which corresponds to $\tau_{1}=\mathrm{T}$ ). Such a curve for a value of $\mathrm{T}^{2}=10$ is shown on Fig. 2 . In this figure as well as all others following, the unit of length on the $x$ and $y$ axis is meter. If the number of switch times on the wheels are exchanged or the controls have opposite signs, curves that are symetric to this one with respect to the $\mathrm{x}$ or $\mathrm{y}$ axis are obtained. Furthermore, if we progressively increase the values of $T$, the curves showing the final locations of the platform continuously expand from the origin toward the higher values of $x$ and $y$. Therefore, it is clear that by adjusting the two parameters ( $\mathrm{T}$ and $\tau_{1}$ ), all points in the first quadrant can be reached by a path involving three switch times. Moreover, if any time optimal paths that reach points in the first quadrant belong to this "three switch times trajectory space," they must be among those leading to points on the first arc of the curves, i.e., that which initiates on the $x$ axis for $\tau_{1}=0$ and sweeps through the first quadrant to the $y$ axis. Samples of these arcs of curves (thereafter referred to as "first arcs") for values of $T$ such that $T^{2}=2,4,6,8$, and 10 are shown on Fig. 3. Sample Cartesian trajectories for paths involving three switch times are displayed in Fig. 4 for the case where $T^{2}=10$. At the end of the paths, both wheels have the same velocity and the Cartesian paths are line segments.

For any point in the first quadrant, we can now use a simple search to directly find the parameters $\left(\tau_{1}, T\right)$ that produce a trajectory of the type shown in Fig. 4 and lead to the desired point. Using the values of the switch times and the transversality condition (see Ref. 10, p. 50) in the approach outlined in Eqs. (42), (47), and (48), the initial conditions for the dual variables can be easily calculated. The equations can then be numerical'y integrated to calculate the dual variables, and to verify that the Cartesian paths leading to "first arcs" are optimal by showing that the dual variables are consistent with the control paths. An example of these results for the point $(0.66,4.03)$ is given in Fig. 5 which displays the time variation of the dual variables $\psi_{3}, \psi_{4}$, and $\psi_{5}$. 


\subsection{TIME-OPTIMAL PATHS WHEN A FINAL CONFIGURATION IS SPECIFIED}

Paths with four switch times can have either two switch times for each wheel ( Iype 22) or one switch time for one wheel and three switch times for the other wheel (Type 13). With the same procedure used in section 5.1 we can fix the final orientation. and find the location of the paths that can reach furthest in the first quadrant while matching the orientation, for given ralues of $\mathrm{T}$.

Figure 6 displays the points that can be reached by Type 22 paths when the orientation is $\phi=0.8$ radians for values of $\mathrm{T}$ such that $\mathrm{T}^{2}=2,4,6,8$ and 10 (the value of amax $=.5 \mathrm{~m} / \mathrm{sec}^{2}$ will remain the same for all figures in this section). Clearly, the paths cannot reach all of the puints in the first quadrant. Figure 7 illustrates the points that can be reached by either Type 22 or Type 13 paths when the orientation is $\psi=0.8$-adians. The Type 13 paths fill the gap that was left by the Type 22 paths. Here too, for the given final orientation, every point in the first quadrant can be reached by adjusing the two parameters ( $T$ and $\tau_{1}$ ). Similar figures can be obtained for varying values of the final orientation. Figure 8 plots the points that can be reached by either type of path with four switch times when the orientation is $\psi=1.57$ radians, while Fig. 9 displays the points that can be reached when the orientation is $\psi=3.14$ radians. In both figures, every point in the first quadrant can be reached by adjusting the two parameters ( $\mathrm{T}$ and $\tau_{1}$ ). Figure 9 has four sets of curves (rather than five like the other figures) because the time required for a pure rotation of 3.14 radians $\left(T^{2}=2.4\right)$ is longer than the first value for the parameter $\mathrm{T}\left(\mathrm{T}^{2}=2\right)$ used in the other figures.

Sample Cartesian trajectories for the paths with four switch times that reach furthest in the first quadrant for a value of $\mathrm{T}^{2}=10$ are displayed in Figs. 10 and 11 for final orientation values of $\phi=0.8$ radians and $\phi=3.14$ radians, respectively. As in Section 5.1, we can use the four switch times to calculate the initial conditions for the dual variables, numerically integrate to calculate the dual variables, and verify that the Cartesian paths of the type shown in Figs. 10 and 11 are optimal by showing that the dual variables are consistent with the control paths. An example of these results for the final configuration $(0.20,3.43,0.80)$ is given in Fig. 12 which shows the time variation of the dual variables $\psi_{4}$ and $\psi_{5}\left(\psi_{3}\right.$ is not constrained in this case). According to Theorem 2 of Pontryagin and with the symetries with respect to the $x$ and $y$ axes, all four-switch-times paths of the type shown in Figs. 10 and 11 are therefore the time optimal paths for the robot to reach any given configuration. 


\subsection{USE OF THE METHOD FOR ROBOT TRAJECTORY PLANNING}

Our objective for implementation on our robots is to determise the ralues of the parameters that define the time optimal trajectory reaching any given final configuration. Given the desired final orientation, Eq. (40) is first used to reduce the number of unknown parameters from three to two. Using a numerical search technique (Powell's method [26]), the two parameters are determined for the given final locatic $(x, y)$ making use of the symetries with respect to the $\mathrm{x}$ and $\mathrm{y}$ axes as appropriate. The switch times can then be calculated and the controls directly sent to the robot actuator servos. Although not required by the method, the corresponding optimal Cartesian trajectories can also be easily calculated if they are needed. As an example of the gain obtained with the tim-optimal trajectories compared with the previous method used to reach a configuration (i.e., rotate, translate, rotate), Table 1 shows the total trajectory times required to reach the point $(3,3)$ at several orientations. T represents the optimal trajectory time, while $S$ is the time required to execute the RTR (rotate, translate, rotate) strategy.

As the orientation increases from 0.80 to 3.14 radians, $T$ increases from 6.18 seconds to 7.04 seconds. The ratio, $R$, of $T$ and $S(R=T / S)$ varies from $81 \%$ to $71 \%$ as the final orientation increases from 0.80 . Thus, the optimal paths are approximately $19 \%$ faster than the RTR path when the final orientation is specified as $\phi=0.80 \operatorname{radians}\left(45^{\circ}\right)$ and $29 \%$ faster when $\phi=1.57$ or 3.14 radians.

Table 1. Values of the optimal half time $(T)$, the half time for rotate-translate-rotate $(S)$, and their ratio $(R)$ for paths that reach the posture $(3,3, \phi)$.

\begin{tabular}{cccc}
\hline $\begin{array}{c}\boldsymbol{T} \\
\text { Radians }\end{array}$ & Seconds & $\begin{array}{c}\mathbf{S} \\
\text { Seconds }\end{array}$ & $\mathbf{R}$ \\
\hline 0.80 & 6.18 & 7.58 & $\mathbf{\%}$ \\
1.57 & 6.36 & 8.92 & 81 \\
3.14 & 7.04 & 10.04 & 71 \\
\hline
\end{tabular}

\section{CONCLUSIONS}

Our objective is to plan time-optimal paths for a mobile platform with two fixed wheels from one configuration to the next in a planar environment without obstacles. We have used Pontryagin's Maximum Principle to prove that, when the maximum acceleration on 
each wheel is bounded. all optimal trajectories are bang-bang. ¿'sing this result. we have proposed a parameterization of the optimal trajectories in a two-dimensional parameter space. We have then shown that paths with three switch times can reach any position. while paths with four switch times can reach any configuration.

We have then followed a five step method to verify that subsets of these paths are timeoptimal. We use the unique two parameters defining a path to calculate the switch times for that path, numerically integrate the control trajectories to calculate the Cartesian paths and some auxilary rariables, use the auxilary rariables to calculate the initial conditions for the dual variables, numerically integrate to calculate the dual variables, and verify that the Cartesian paths are optimal by showing that the dual variables are consistent with the optimal control paths as required by the maximum principle. We have then discussed how the method can be utilized in conjunction with search techniques to directly find the time-optimal controls allowing a platform to reach any arbitrary configuration. For an illustrative value of $0.5 \mathrm{~m} / \mathrm{sec}^{2}$ for the bound on acceleration, comparative examples indicate that time-optimal paths can be up to $29 \%$ faster than rotate-translate-rotate paths.

\section{REFERENCES}

1. R. A. Brooks, "Elephants Don't Play Chess," Robotics and Autonomous Systems 6(1 \& 2), 3-15 (1990).

2. R. C. Arkin, "Integrating Behavioral, Perceptual, and World Knowledge in Reactive Navigation," Robotics and Autonomous Systems 6(1 \& 2), 105-122 (1990).

3. Y. Koren and J. Borenstein, "Potential Field Methods and Their Inhererit Limitations for Mobile Robot Navigation," Proc. IEEE International Conjerence on Robotics and Automation, 1398-1404 (1991).

4. G. L. Blaisdell, "Performance of an Omnidirectional Wheel on Snow and Ice," Naval Engineers Journal 103(1), 34-41 (1991).

5. S. M. Killough and F. G. Pin, "A Fully Omnidirectional Wheeled Assembly for Robotic Vehicles," Trans. Am. Nucl. Soc. 61 425-426 (1990).

6. H. A. Vasseur, F. G. Pin, and J. R. Taylor, "Navigation of a Car-Like Mobile Robot Using a Decomposition of the Environment in Convex Cells," Proc. IEEE Internationa? Conference on Robotics and Automation, 1496-1502 (1991). 
7. G. Giralt, R. Chatila, and M. Vaisset, "An Integrated Navigation and Motion Control System for Autonomous Multisensory Mobile Robots," Robotics Research: The First International Symposium, M. Brady and R. P. Paul, eds., MIT Press, Cambridge, Massachusetts, 1984.

8. Y. Kanayama and B. I. Hartman, "Smooth Local Path Planning for Autonomous Vehicles." Proc. IEEE International Conference on Robotics and Auiomation, 1265-1270 (1989).

9. C. R. Weisbin, G. de Saussure, J. R. Einstein, F. G. Pin, and E. Heer, "Autonomous Mobile Robot Navigation and Learning," Computer 22(6), 29-35 (June 1989).

10. L. S. Pontryagin et al., L. S. Pontryagin Selected Works, Volume 4: The Mathematical Theory of Optimal Processes, Gordon and Breach Science Publishers, New York (1986).

11. M. E. Kahn and B. Roth, "The Near-Minimum-Time Control of Open-Loop Articulated Kinematic Chains," ASME Journal of Dynamic Systems, Measurement, and Control 93(3), 164-172 (1971).

12. M. Niv and D. M. Auslander, "Optimal Control of a Robot with Obstacles," Proc. of the American Control Conference, 280-287 (1984).

13. B. K. Kim and K. G. Shin, "Suboptimal Control of Industrial Manipulators with a Weighted Minimum Time-Fuel Criterion," IEEE Transactions on Automatic Control 30(1), 1-10 (1985).

14. A. Weinreb and A. E. Bryson, "Optimal Control of Systems with Hard Control Bounds," IEEE Transactions on Automatic Control 30(11), 1135-1138 (1985).

15. Y. Nakamura and H. Hanafusa, "Optimal Redundancy Control of Robot Manipulators," Int. J. Robotics Research 6(1), 32-42 (1987).

16. J. E. Bobrow, "Optimal Robot Path Planning Using the Minimum-Time Criterion," IEEE J. of Robotics and Automation 4(4), 443-450 (1988).

17. M. Yamamoto and A. Mohri, "Planning of Quasi-Minimum Time Trajectories for Robot Manipulators (Generation of a Bang-Bang Control)," Robotica 7, 43-47 (1989).

13. A. Ailon and G. Langholtz, "On the Existence of Time-Optimal Control of M Mechanical Manipulators, Journal of Optimization Theory and Applications 46(1), 1-21 (1985).

19. J. Wen, "On Minimum Time Control for Robotic Manipulators," Recent Trends in Robotics: Modeling, Control, and Education, North-Holland, New York, 283-292 (1986).

20. L. G. Van Willigenburg, "First Order Controllability and the Time Optimal Control Problem for Rigid Articulated Arm Robots with Friction," Int. J. Control 51(6), 1159-1171 (1990). 
21. Y. Chen and A. A. Desrochers. "A Proof of the Structure of the Minimum-Time Control Law of Robotic Manipulators Uising a Hamiltonian Formulation," IEEE Transactions on Robotics and Automation 6(3), 388-393 (1990).

22. H. P. Geering, et al., "Time-Optimal Motions of Robots in Assembly Tasks," IEEE Transactions on Automatic Control 31(6), 512-518 (1986).

23. S. N. Osipov and A. M. Formal'skii, "The Problem of the Time-Optimal Turning of a Manipulator," Applied Mathematics and Mechanics 52(6), 725-731 (1988).

24. A. M. Formal'skii and S. N. Osipov, "On the Problem of the Time-Optimal Manipulator Arm Turning," IEEE Transactions on Automatic Control 35(6), 714-719 (1990).

25. P. Jacobs, J. P. Laumond, and A. Rege, "Non-Holonomic Motion Planning for HilareLike Mobile Robots," F'roc. of the International Symposium on Intelligent Robotics, 338-347 (1991).

26. W. H. Press et al., Numerical Recipis in C: The Art of Scientific Computing, Cambridge, UK: Cambridge University Press, 1988. 


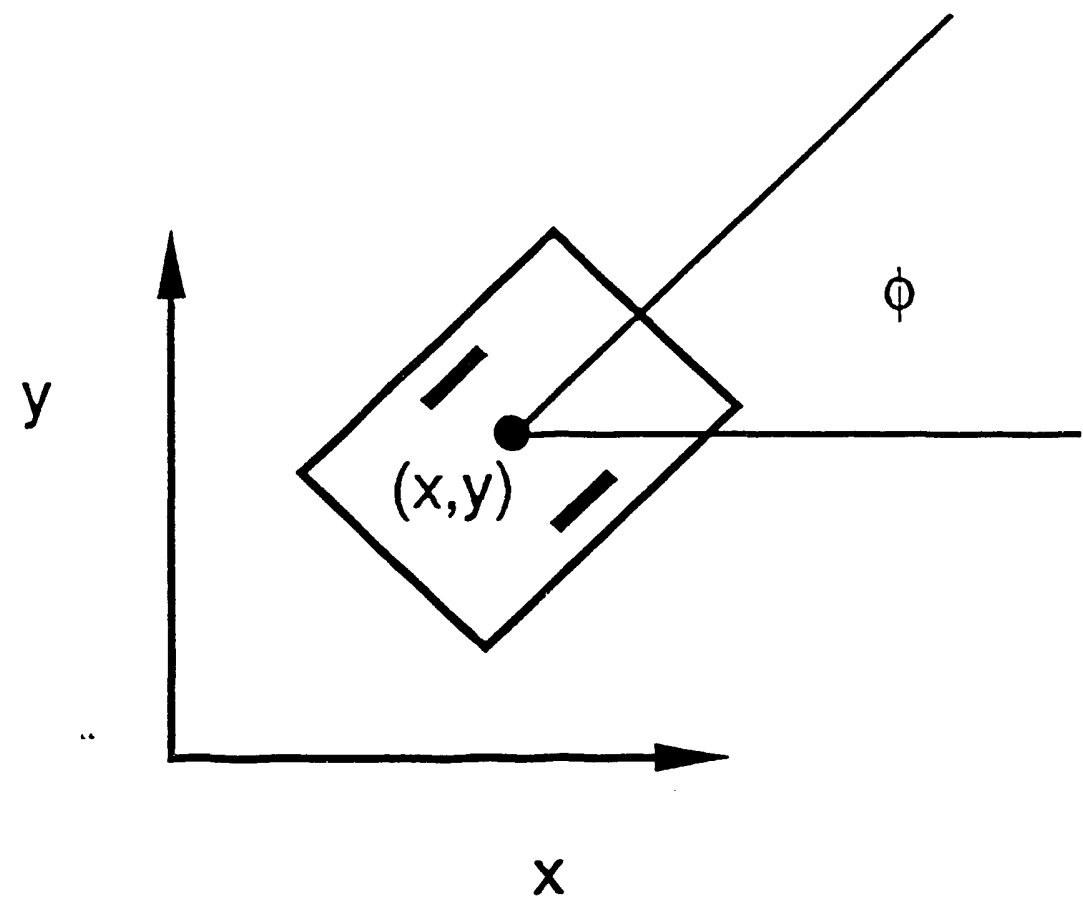

Fig. 1. The configuration of the platform is defined by the position $(x, y)$ and orientation $(\phi)$. 


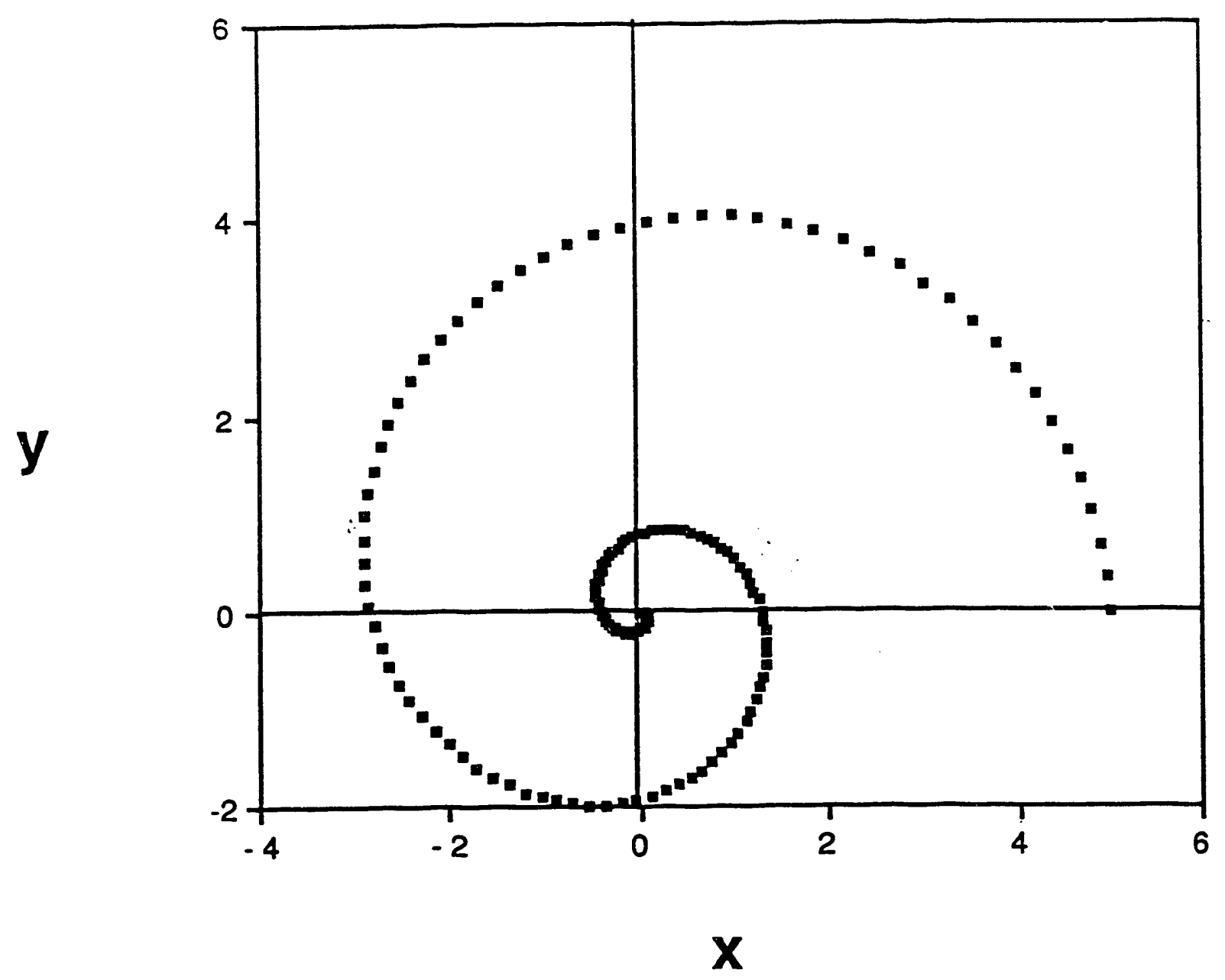

Fig. 2. Location of the final platform positions for paths involving one switch time on the right wheel and two on the left wheel, for a constant value of $\mathrm{T}^{2}=10$. 


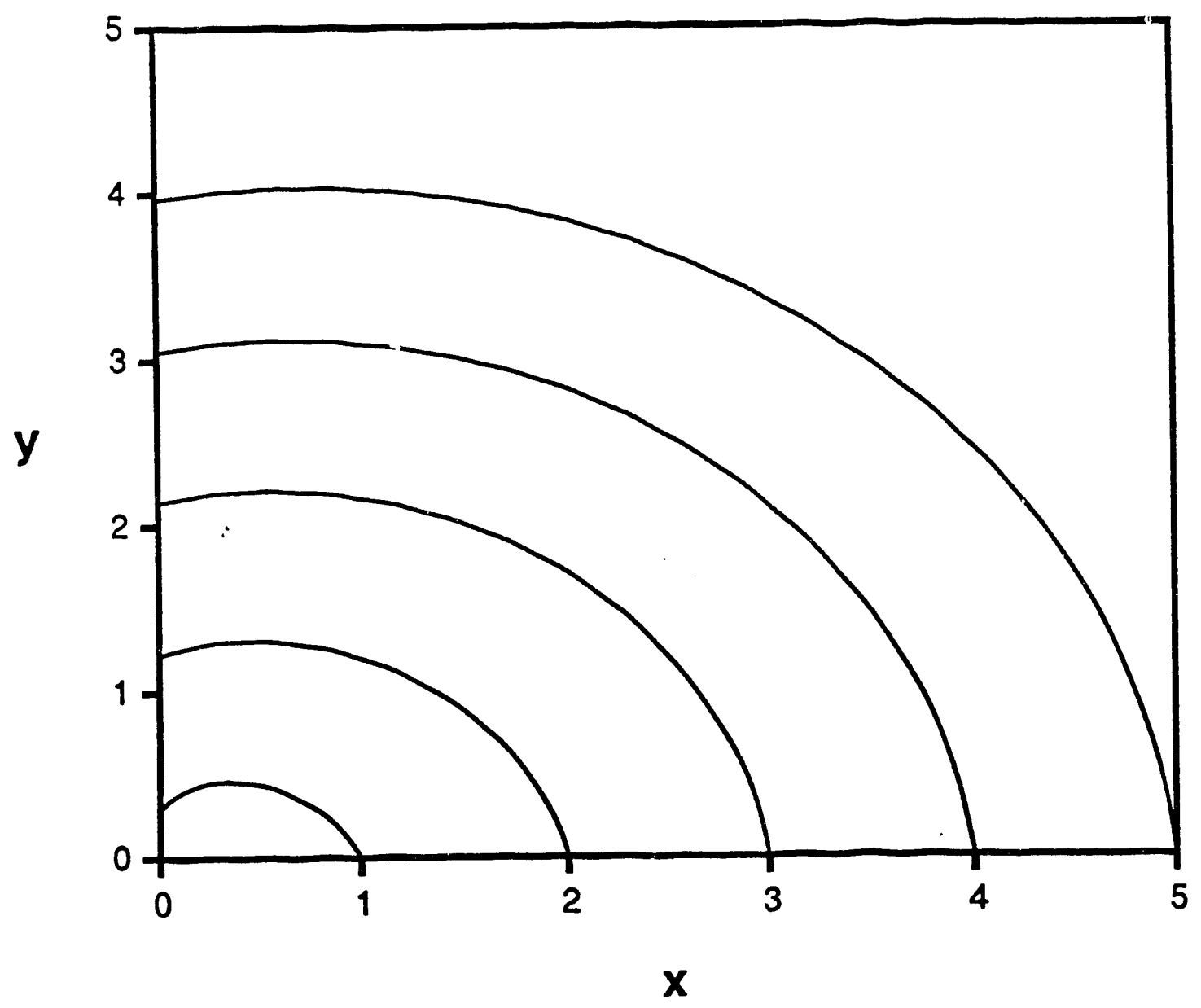

Fig. 3. Curves showing the location of points in the first quadrant that can be reached in minimum time by paths with three switch times for values of $T^{2}=2,4,6,8$ and 10 . 


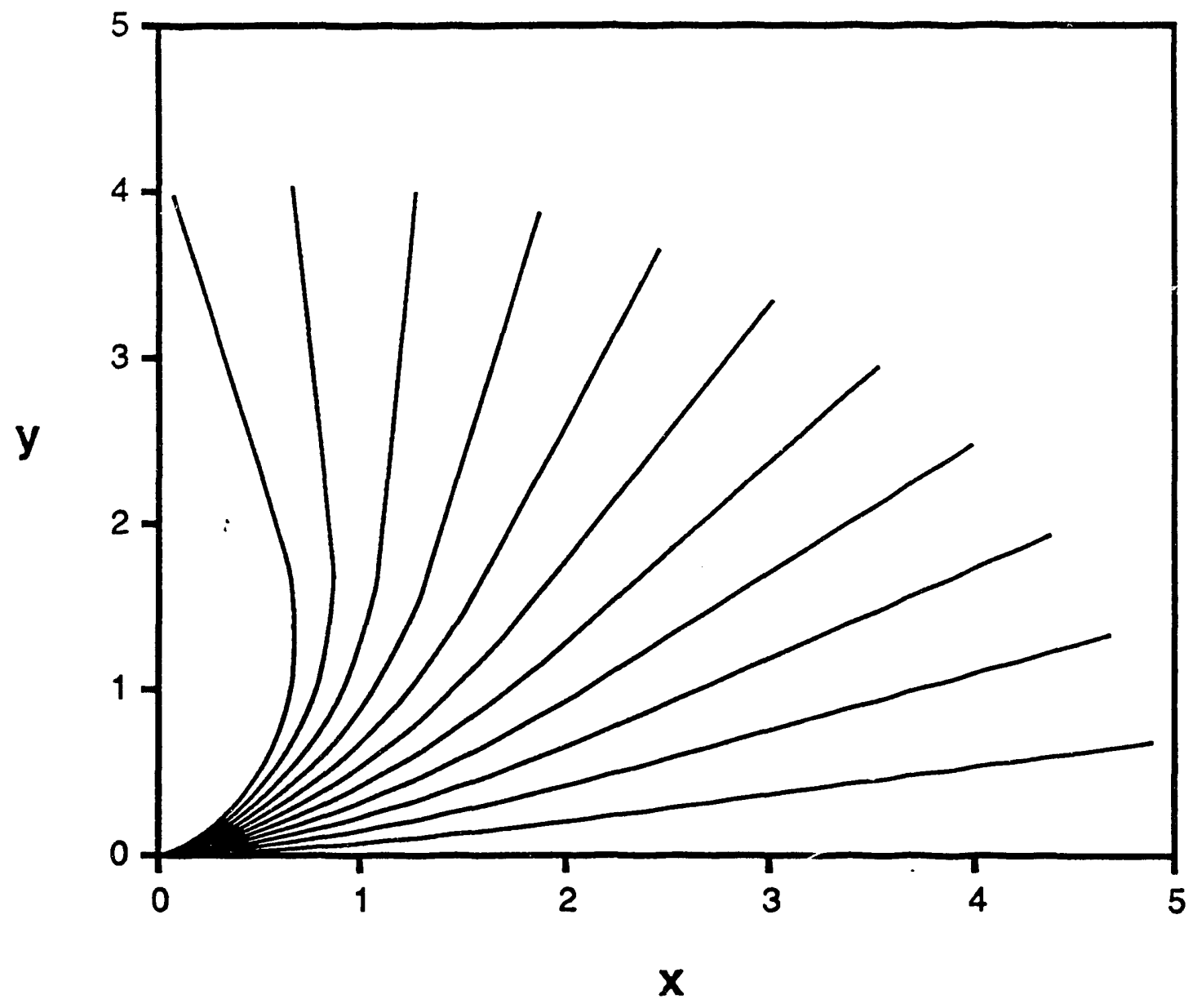

Fig. 4. Cartesian trajectories for paths with three switch times for a value of $T^{2}=10$. 


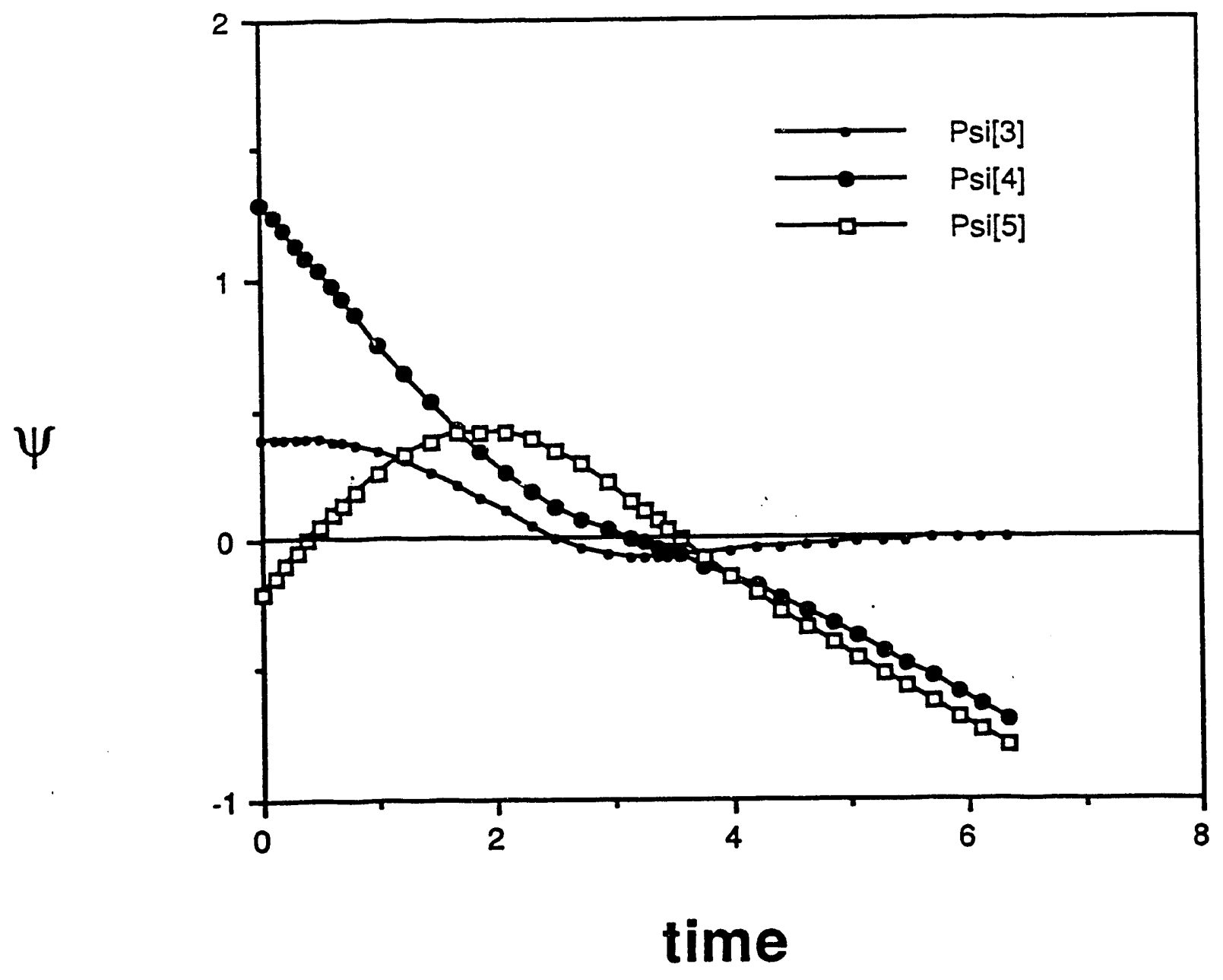

7ig. 5. Dual variables for the time optimal path leading to point $(0.66,4.03)$. 


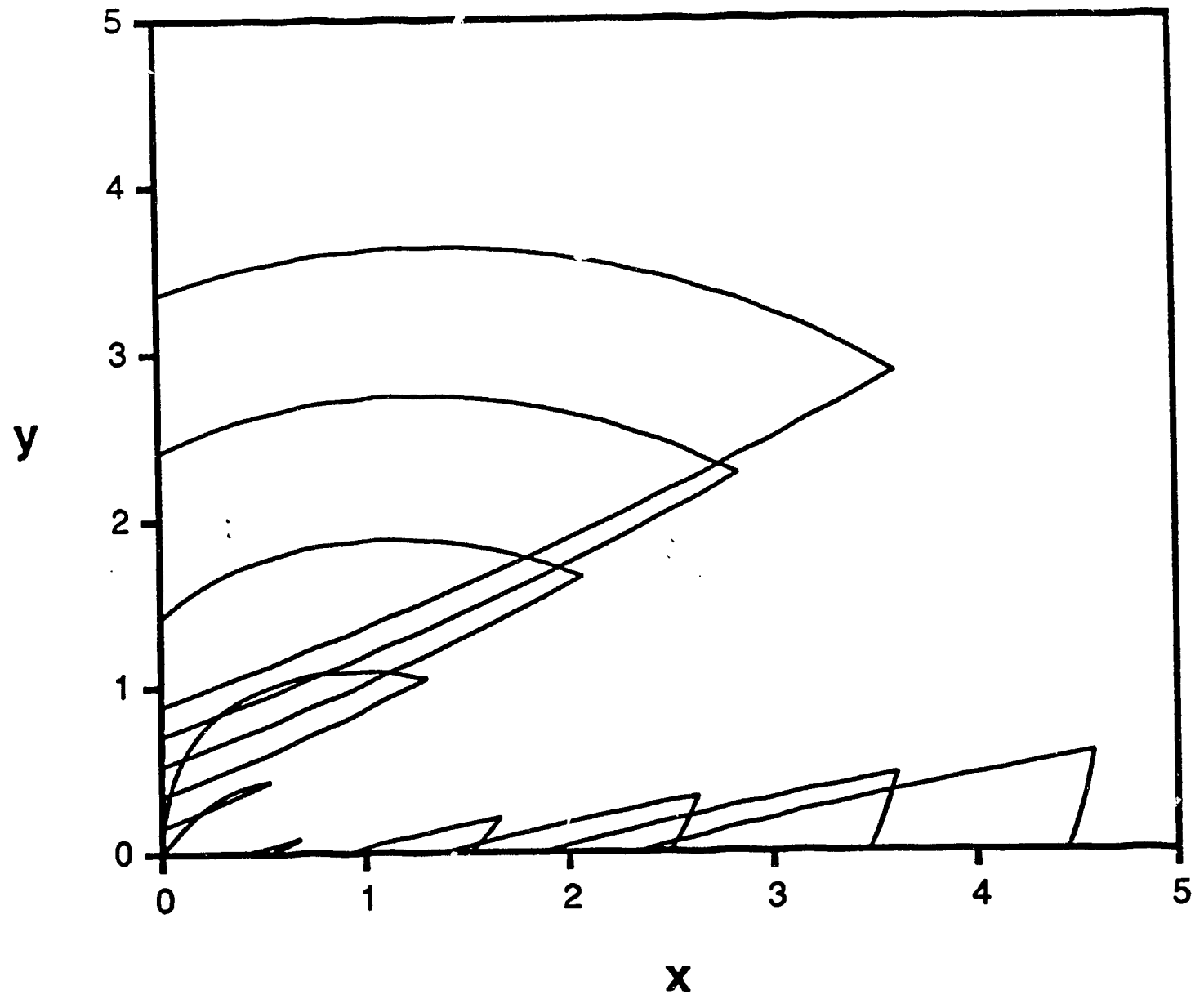

Fig. 6. Points in the first quadrant that can be reached by a type 22 path with four switch times when the final orientation is 0.8 radians. Curves are for values of $\mathrm{T}^{2}=2,4,6,8$ and 10 . 


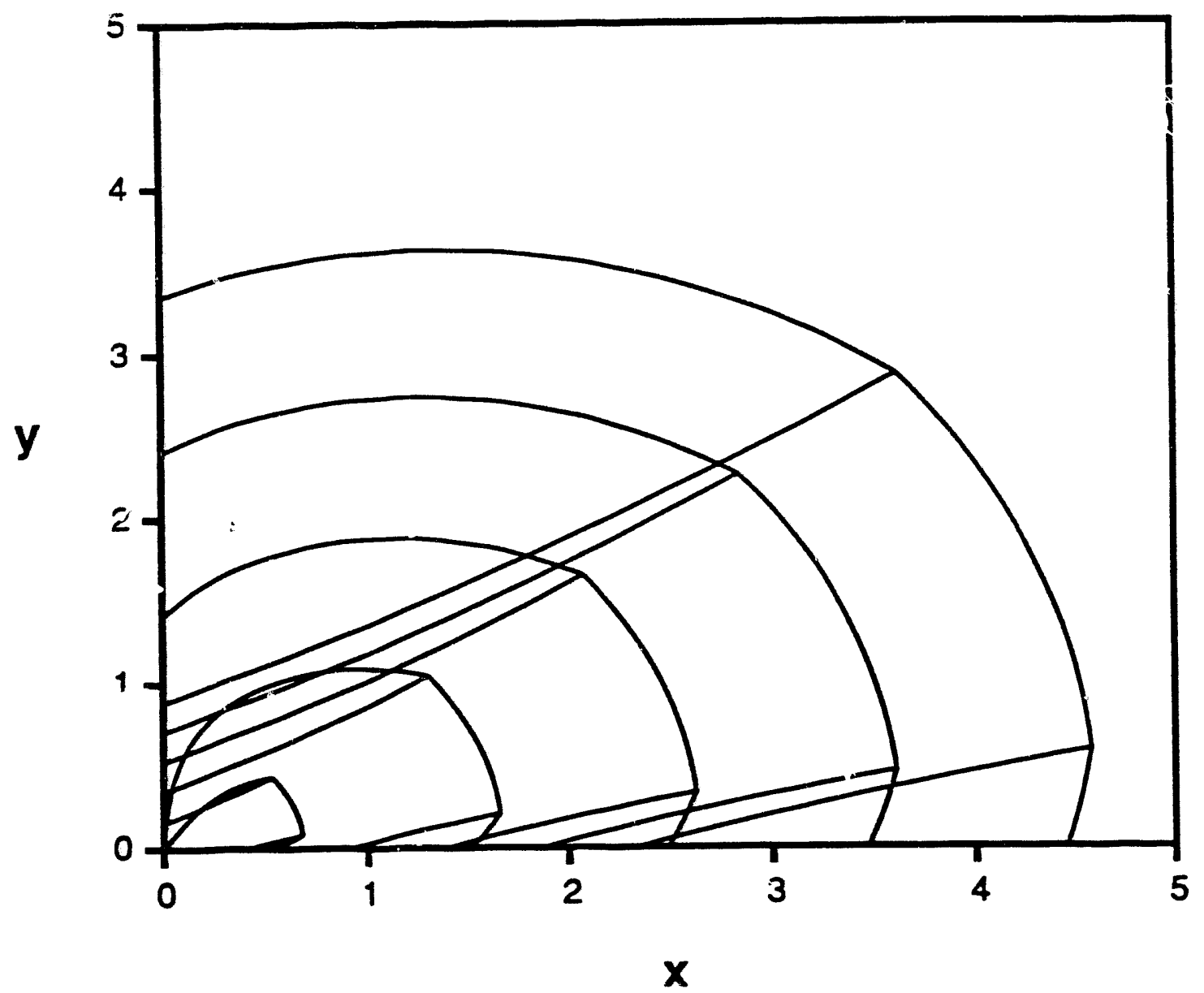

Fig. 7. Points in the first quadrant that can be reached by paths with four switch times when the final orientation is 0.8 radians. Curves are for values of $T^{2}=2,4,6,8$ and 10 . 


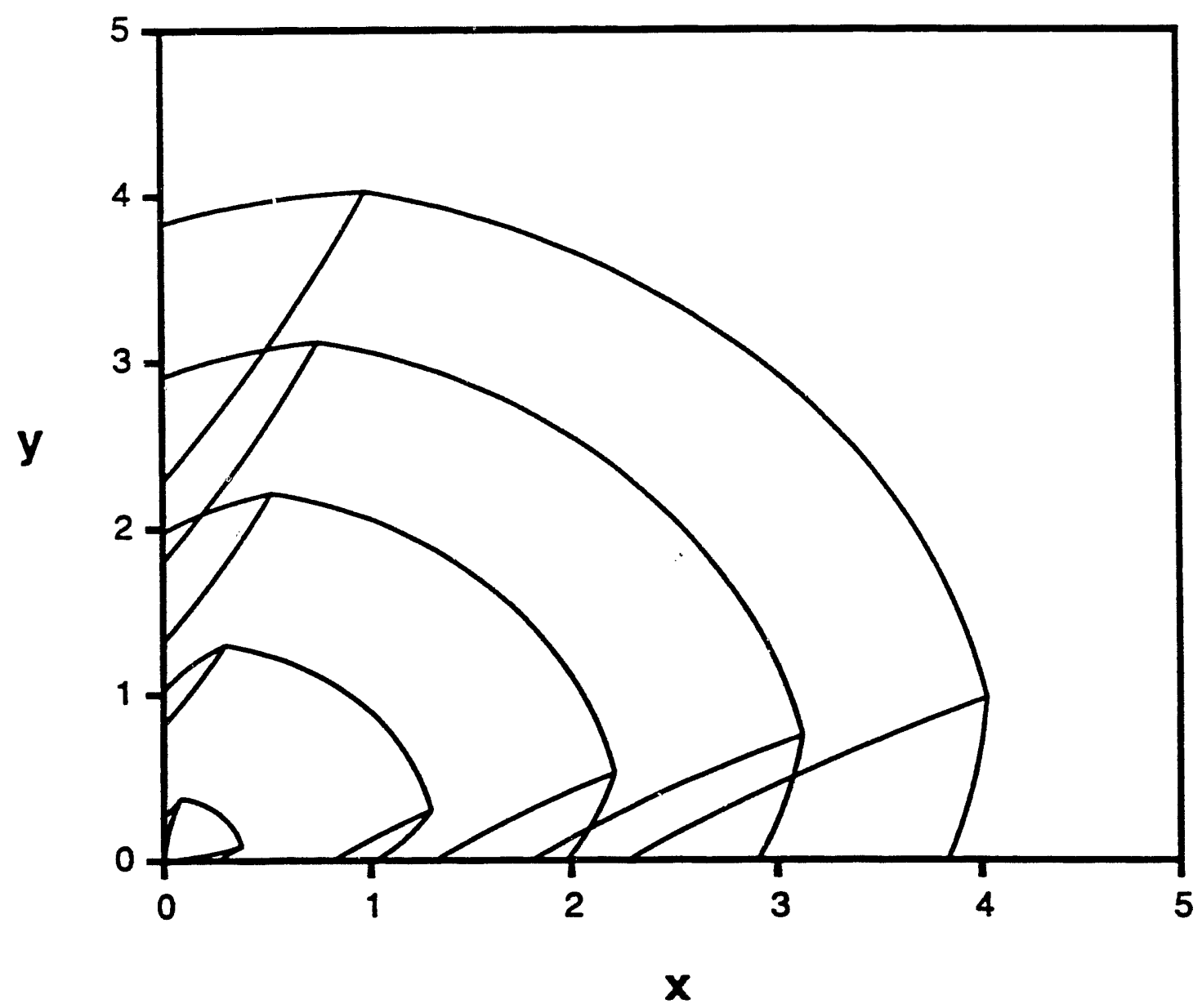

Fig. 8. Points in the first quadrant that can be reached by paths with four switch times when the final orientation is 1.57 radians. Curves are for values of $T^{2}=2,4,6,8$ and 10 . 


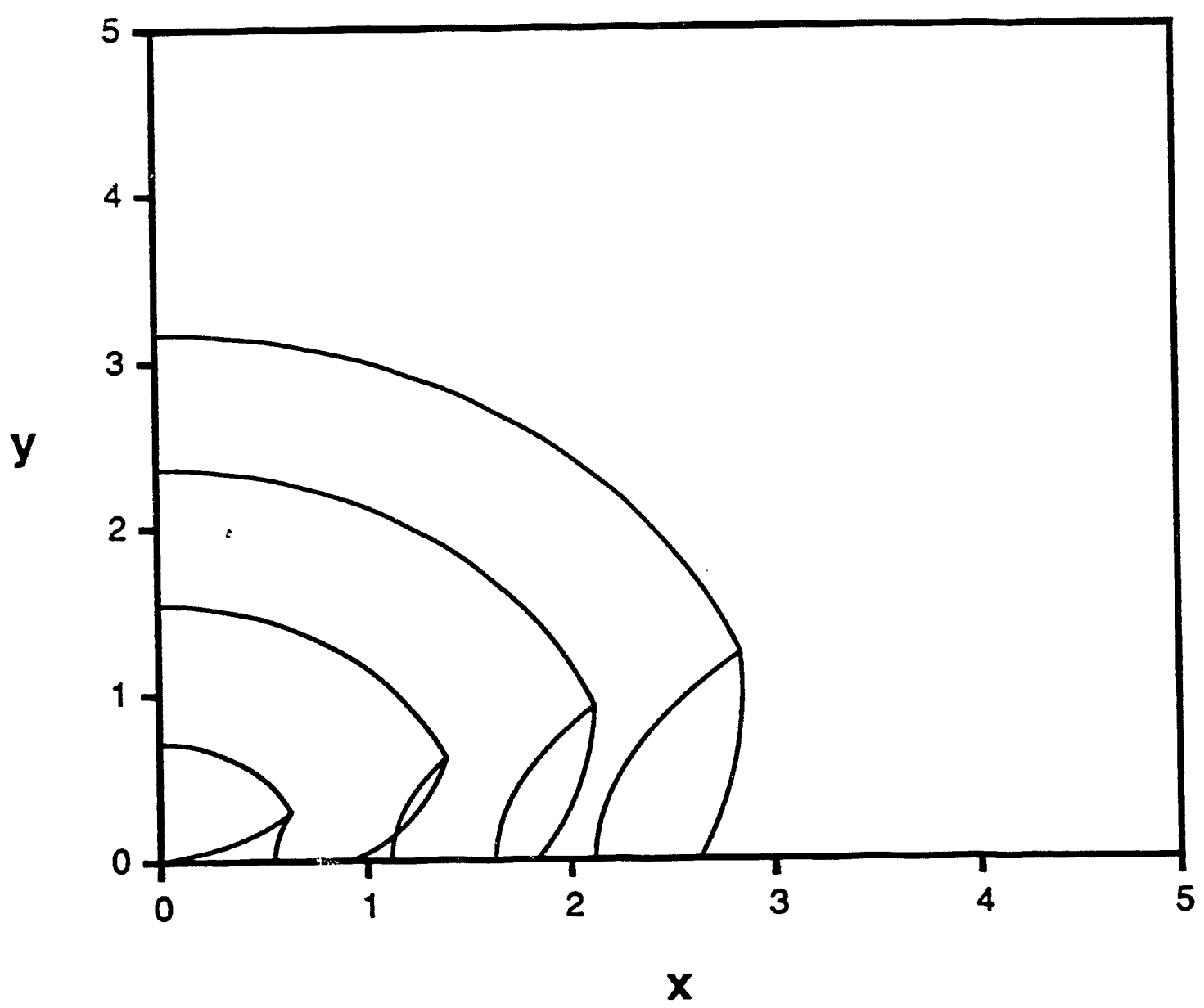

Fig. 9. Points in the first quadrant that can be reached by paths with four switch times when the final orientation is 3.14 radians. Curves are for values of $T^{2}=4,6,8$ and 10 . 


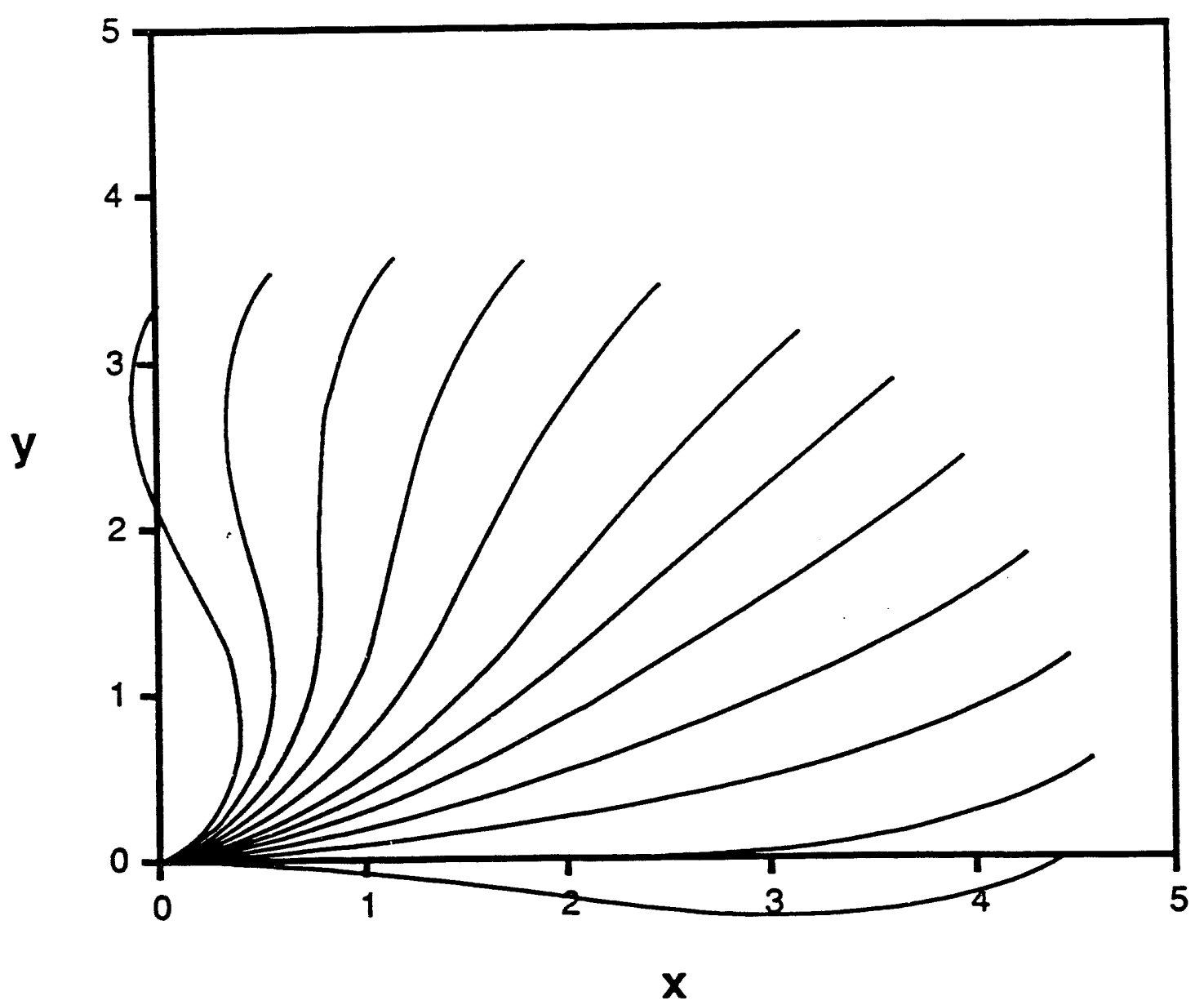

Fig. 10. Cartesian trajectories for paths with four switch times when the final orientation is 0.8 radians and $T^{2}=10$. 


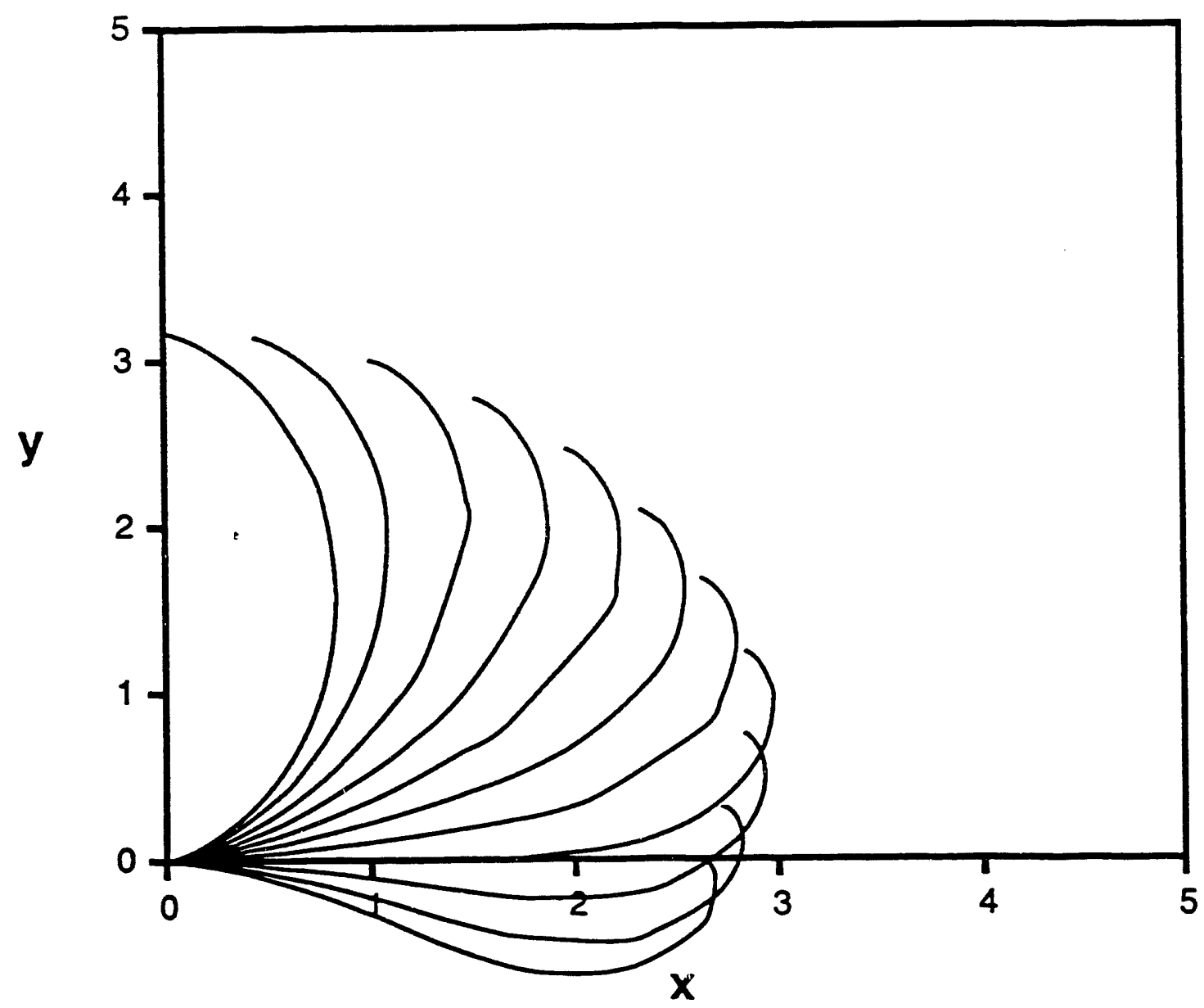

Fig. 11. Cartesian trajectories for paths with four switch times when the final orientation is 3.14 radians and $\mathrm{T}^{2}=10$. 


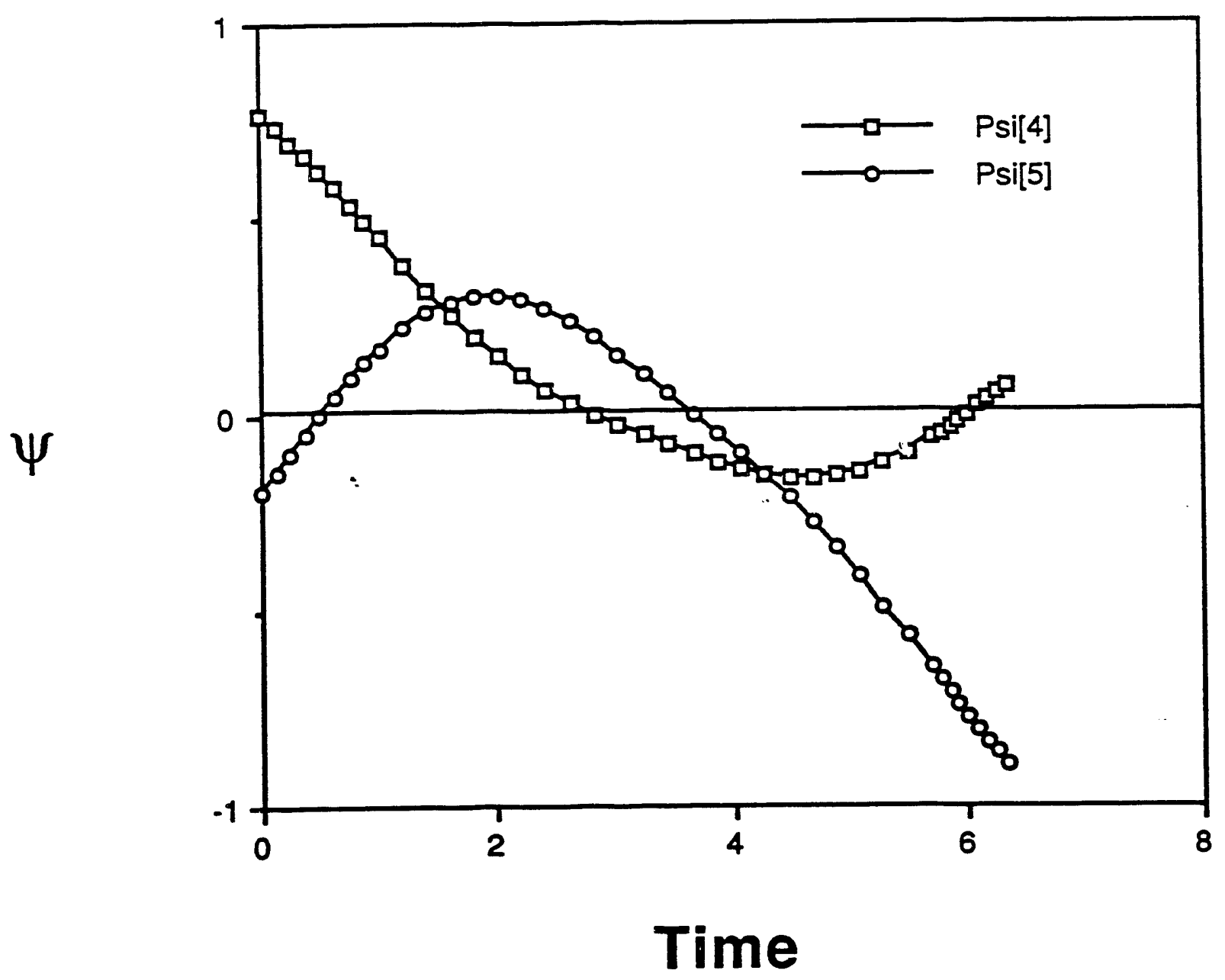

Fig. 12. Dual variables for the time-optimal path reaching the configuration $(0.20$, $3,43,0.80)$. 

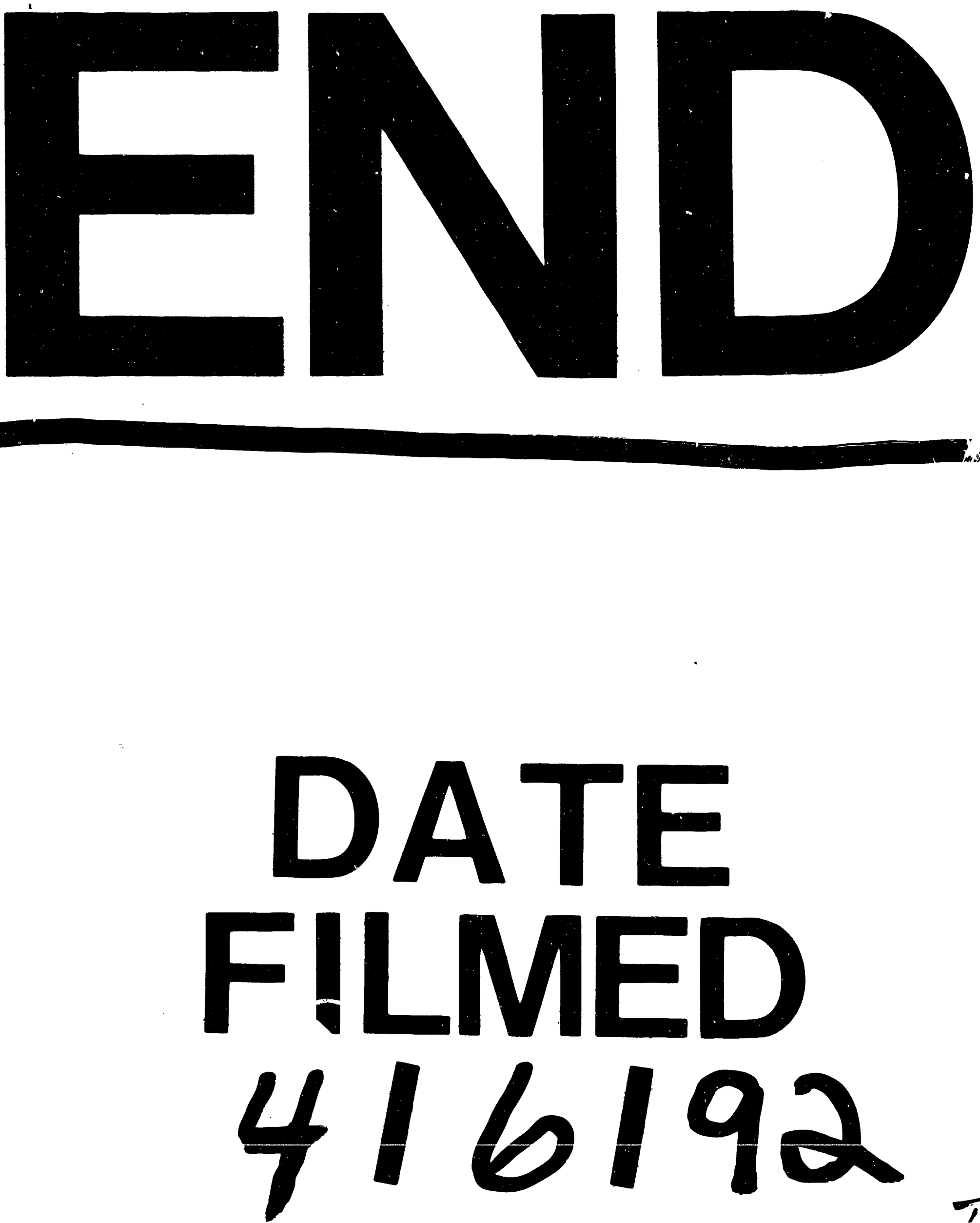

I. 
